\title{
Mitrofanovite $\mathrm{Pt}_{3} \mathrm{Te}_{4}$ : A Topological Metal with Termination-Dependent Surface Band Structure and Strong Spin Polarization
}

Jun Fujii, ${ }^{\nabla}$ Barun Ghosh, ${ }^{\nabla}$ Ivana Vobornik, ${ }^{*}, \nabla$ Anan Bari Sarkar, Debashis Mondal, Chia-Nung Kuo, François C. Bocquet, Lixue Zhang, Danil W. Boukhvalov, Chin Shan Lue, Amit Agarwal,* and Antonio Politano*

S1 Supporting Information

ABSTRACT: Due to their peculiar quasiparticle excitations, topological metals have high potential for applications in the fields of spintronics, catalysis, and superconductivity. Here, by combining spin- and angle-resolved photoemission spectroscopy, scanning tunneling microscopy/spectroscopy, and density functional theory, we discover surface-termination-dependent topological electronic states in the recently discovered mitrofanovite $\mathrm{Pt}_{3} \mathrm{Te}_{4}$. Mitrofanovite crystal is formed by alternating, van der Waals bound layers of $\mathrm{Pt}_{2} \mathrm{Te}_{2}$ and $\mathrm{PtTe}_{2}$. Our results demonstrate that mitrofanovite is a topological metal with terminationdependent (i) electronic band structure and (ii) spin texture. Despite their distinct electronic character, both surface terminations are characterized by electronic states exhibiting strong spin polarization with a node at the $\Gamma$ point and sign reversal across the $\Gamma$ point, indicating their topological nature and the possibility of realizing two distinct electronic configurations (both of them with topological features) on the surface of the same material.

KEYWORDS: topological metals, surface states, STM/STS, spintronics, ARPES

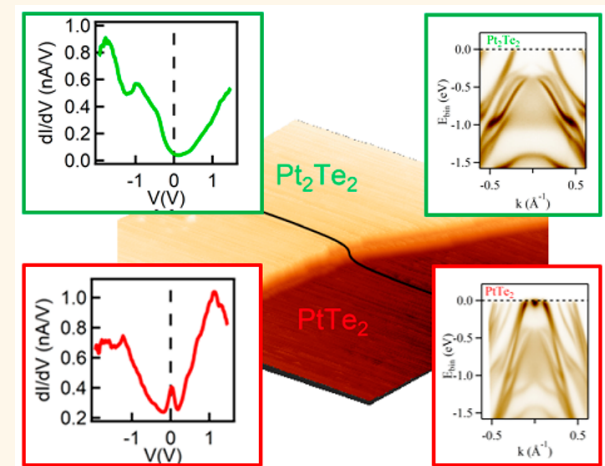

\section{INTRODUCTION}

Topological metals are materials with nontrivial band crossings or band inversions near the Fermi energy, giving rise to peculiar quasiparticle excitations. ${ }^{1-8}$ They can be classified based on the dimensionality and degeneracy of their band crossings. ${ }^{9}$ Prominent examples include Dirac, ${ }^{10}$ Weyl, ${ }^{11}$ nodal-line, ${ }^{12}$ and nodal-surface metals. ${ }^{9}$ In topological metals, bulk superconductivity can also coexist with topologically nontrivial states, as demonstrated for $\mathrm{PbTaSe}_{2}{ }^{13}$ enabling the intriguing perspective of Majorana Fermions in solid-state physics. Furthermore, the heterostructures of topological metals are being pursued as suitable candidates for potential applications in quantum computing. ${ }^{14}$ The fascinating technological capabilities of topological metals are also confirmed by recent reports indicating their superior efficiency in catalytic reactions and hydrogen production, as demonstrated for the case of the Pt-based alloys PtAl and PtGa. ${ }^{15}$

Among the various families of materials showing gapless Dirac Fermions, the transition-metal dichalcogenide $\mathrm{TMX}_{2}$ $(\mathrm{TM}=\mathrm{Pd}, \mathrm{Pt} ; \mathrm{X}=\mathrm{Se}, \mathrm{Te})$, crystallizing in the same structure as the naturally occurring mineral "moncheite", ${ }^{16}$ was demonstrated to host type-II Dirac fermions, ${ }^{17}$ with application capabilities in plasmonics, ${ }^{10}$ catalysis, ${ }^{18}$ nanoelectronics, ${ }^{19}$ and wearable electronics. ${ }^{20}$ These properties can be tuned by varying (i) the position of the Fermi level with respect to the degenerate Dirac (or Weyl/nodal line) point and (ii) the strength of the spin-orbit coupling.

Mitrofanovite $\mathrm{Pt}_{3} \mathrm{Te}_{4}$, belonging to the "moncheite" family of materials with trigonal space group $R \overline{3} m$ (No. 166), has recently been discovered as a natural mineral in the Kola Peninsula, Russia, ${ }^{21}$ and in different zones in the Canadian Shield. ${ }^{22}$ Its atomic structure is constituted by alternating layers of hexagonal $\mathrm{PtTe}_{2}$ and $\mathrm{Pt}_{2} \mathrm{Te}_{2}$ (or $\mathrm{PtTe}$ ) which are stacked along the vertical direction and held together by weak van der Waals interactions. Very recently, mitrofanovite has been demonstrated to be an efficient and stable catalyst for hydrogen evolution reaction (HER) with an overpotential of

Received: June 3, 2021

Accepted: August 10, 2021

Published: September 2, 2021 
a)

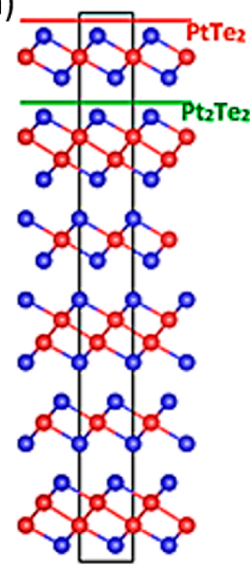

b)

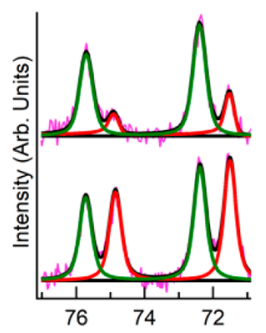

c) d)

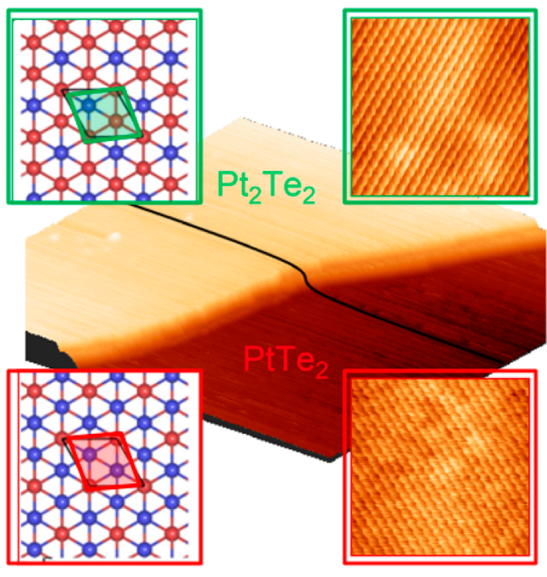

e)
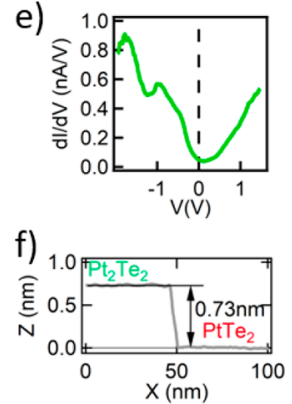

g)

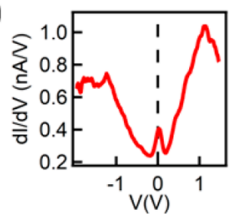

Figure 1. (a) Side view of the $\mathrm{Pt}_{3} \mathrm{Te}_{4}$ crystal structure with the two different cleavage planes- $\mathrm{Pt}_{2} \mathrm{Te}_{2}$ (green) and $\mathrm{PtTe}_{2}$ (red) terminations are identified. (b) Pt-4f XPS spectra for the $\mathrm{Pt}_{2} \mathrm{Te}_{2}$ (top) and $\mathrm{PtTe}_{2}$ (bottom) terminations, respectively. (c) Hexagonal LEED pattern obtained on both terminations. (d) 3D STM topographic image $(100 \mathrm{~nm} \times 100 \mathrm{~nm})$ showing the adjacent terraces of different terminations separated by a step; the four insets framed green (red) are the top view of the crystal structure and the atomically resolved STM image (7 nm $\times 7 \mathrm{~nm}$ ) for the $\mathrm{Pt}_{2} \mathrm{Te}_{2}\left(\mathrm{PtTe}_{2}\right)$ terminations. (e and g) STS spectra measured on the $\mathrm{Pt}_{2} \mathrm{Te}_{2}$ and the $\mathrm{PtTe}_{2}$ terraces. We observe a zero-bias peak only on the $\mathrm{PtTe}_{2}$ termination. (f) Height profile along the black line in panel $\mathrm{d}$.

$39.6 \mathrm{mV}$ and a Tafel slope of $32.7 \mathrm{mV} / \mathrm{dec}$ together with a high current density exceeding $7000 \mathrm{~mA} / \mathrm{cm}^{2} .^{23}$

Here, we explore the electronic properties of $\mathrm{Pt}_{3} \mathrm{Te}_{4}$ by means of scanning tunneling microscopy (STM)/spectroscopy (STS) and spin- and angle-resolved photoemission spectroscopy (spin-ARPES) in conjugation with density functional theory (DFT). We demonstrate that mitrofanovite is a topological metal hosting spin-polarized surface states. Interestingly, we find that $\mathrm{Pt}_{3} \mathrm{Te}_{4}$ has two distinct surface terminations with radically different electronic properties. These distinct terminations are observed at different terraces on the same face of the cleaved crystal. Despite differences in the corresponding electronic band structure, both surface terminations host spin-polarized states, exhibiting typical polarization reversal across the zone center, characteristic of spin-momentum locking. Thus, mitrofanovite offers termination-dependent electronic and surface properties enabling tunable device functionalities for nanoelectronics, spintronics, optoelectronics, and plasmonic applications.

\section{RESULTS AND DISCUSSION}

Identification of Two Distinct Surface Terminations. The unit cell of $\mathrm{Pt}_{3} \mathrm{Te}_{4}$ is composed of alternating blocks of $\mathrm{PtTe}_{2}$ and $\mathrm{Pt}_{2} \mathrm{Te}_{2}$ units stacked vertically, as depicted in Figure 1a for the side view and in the red and green boxes in the inset of Figure 1d for top views. Specifically, in the $\mathrm{PtTe}_{2}$ subunit, one atomic Pt layer is sandwiched between the two Te layers. Contrariwise, in the $\mathrm{Pt}_{2} \mathrm{Te}_{2}$ subunit, two layers of $\mathrm{Pt}$ atoms are sandwiched between two Te layers. For such a crystal structure, two cleavage planes are feasible, resulting either in (i) a $\mathrm{PtTe}_{2}$ termination (marked by a red line in Figure 1a) or (ii) a $\mathrm{Pt}_{2} \mathrm{Te}_{2}$ termination (green line in Figure 1a). For the sake of completeness, also a PtTe termination is in principle feasible. However, as the energy per surface unit for the PtTeterminated slab is more than $1 \mathrm{eV}$ larger than for the $\mathrm{PtTe}_{2}$ and $\mathrm{Pt}_{2} \mathrm{Te}_{2}$-terminated slab, this type of termination can be excluded from further discussion.
Thus, the existence of two distinct interfaces at the surface of the mitrofanovite crystal enables tunable electronic, spintronic, and plasmonic properties of the electrode contact for exploitation in micro- and nanoelectronics.

By means of high-resolution X-ray spectroscopy (XPS) on an as-cleaved sample, we identified two distinct spectral components in the region of $\mathrm{Pt}-4 \mathrm{f}$ core levels. In our experimental configuration (low-energy photons of $120 \mathrm{eV}$ in normal emission geometry), the probing depth is $4.2 \pm 0.1 \AA$, based on the effective attenuation length according to the Tanuma-Powel-Penn (TPP-2M) formula. ${ }^{24}$ This is comparable with the size of the single $\mathrm{Pt}_{2} \mathrm{Te}_{2}$ subunits (4.02 $\AA$ ). Therefore, we expect that the two surfaces with different terminations will be reflected by different Pt binding energies (BE).

Consistent with our expectations, we observe a splitting in the Pt-4f core levels, with $J=7 / 2$ components having a $\mathrm{BE}$ of 71.5 and $72.4 \mathrm{eV}$. To assign the two spectral components, we computed core-level shifts based on the charge distribution in the $\mathrm{Pt}_{2} \mathrm{Te}_{2}$ subunit. We found that, in the $\mathrm{Pt}-\mathrm{Te}$ bonds, the charge on the Pt sites is reduced by 0.363 electrons compared to the $\mathrm{Pt}-\mathrm{Pt}$ bonds. The intensity ratio between the two spectral components is $1.0 \pm 0.1$ in the $\mathrm{Pt}_{2} \mathrm{Te}_{2}$-termination. Concerning the $\mathrm{PtTe}_{2}$-terminated surface, we observe a suppression of the $\mathrm{Pt}-4 \mathrm{f}$ component related to $\mathrm{Pt}-\mathrm{Pt}$ bonds up to $75 \%$. The total quenching was not obtained, due to the combined effect of the limited spatial resolution (beam size $150 \times 50 \mu \mathrm{m}^{2}$ ) and the insufficient probing depth to selectively choose the contributions from the $\mathrm{PtTe}_{2}$ subunit only, whose size is $2.71 \AA$.

The two different surface terminations were further identified by means of scanning tunneling microscopy (STM) experiments. The three-dimensional STM topographic image $\left(100 \times 100 \mathrm{~nm}^{2}\right)$ in Figure 1d highlights the adjacent large terraces separated by a step on a cleaved surface of mitrofanovite. The height profile shown in Figure $1 \mathrm{f}$ of the step in Figure 1d reveals a stacking of $0.73 \mathrm{~nm}$, with respect to the $\mathrm{Pt}_{2} \mathrm{Te}_{2}$ termination. This stacking distance is consistent with 


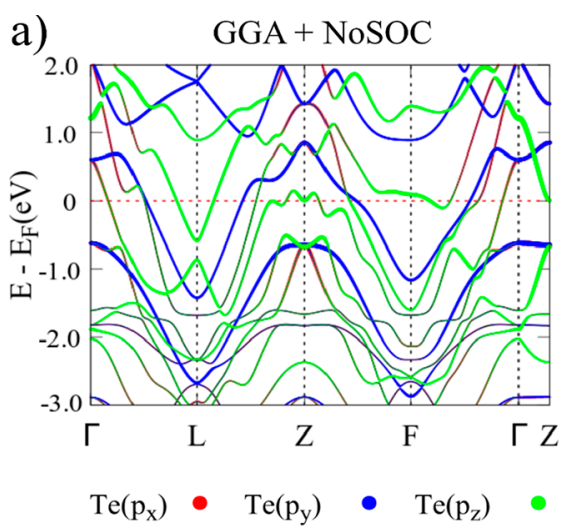

b)

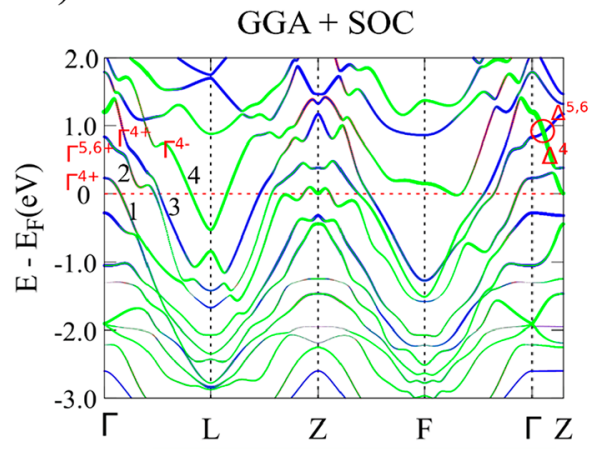

c)

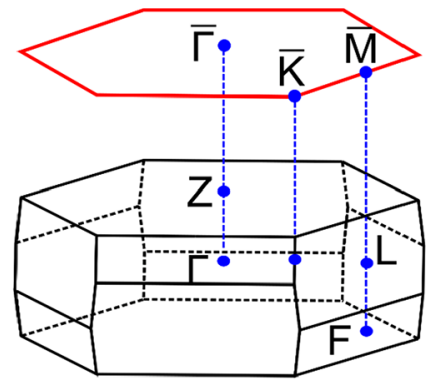

d)
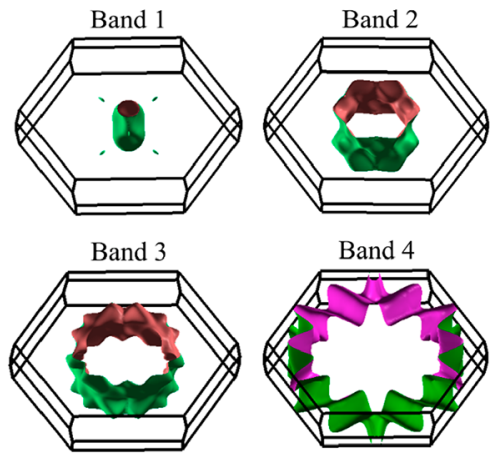

Figure 2. Calculated bulk band structure of $\mathrm{Pt}_{3} \mathrm{Te}_{4}$ along with the orbital character (a) excluding and (b) including the spin-orbit coupling. The irreducible representations of the bands at the $\Gamma$ point are shown. The Dirac point along the $\Gamma-Z$ direction is highlighted in the red circle. There are four bands crossing the Fermi level-marked as bands 1, 2, 3, and 4. Bands 1, 2, and 3 primarily result in hole pockets, while band 4 mainly leads to an electron pocket. (c) The bulk and the (111) surface projected Brillouin zone of the primitive cell with the high-symmetry points marked explicitly. (d) Fermi surfaces for four bands crossing the Fermi surface, clearly showing the presence of several hole and electron pockets arising from bands 1-4.

the distance between the $\mathrm{Pt}_{2} \mathrm{Te}_{2}$ (depicted in the left greenframed inset of Figure 1d) and $\mathrm{PtTe}_{2}$ (depicted in the left redframed inset of Figure 1d) subunits shown schematically in Figure 1a. The corresponding atomic resolution STM image of the $\mathrm{Pt}_{2} \mathrm{Te}_{2}\left(\mathrm{PtTe}_{2}\right)$ terrace is shown in the right inset of Figure $1 \mathrm{~d}$, within the green (red) box.

To compare the local electronic density of states (LDOS) on the two distinct surface terminations, we measured the differential conductance (or $\mathrm{dI} / \mathrm{dV}$ ) plots with STS on both the $\mathrm{Pt}_{2} \mathrm{Te}_{2}$ (Figure 1e, green curve) and the $\mathrm{PtTe}_{2}$ (Figure 1g, red curve) terminations, respectively. We find that the two different terminations show very different $\mathrm{dI} / \mathrm{dV}$ characteristics over a wide range of the chemical potential (from -2.0 to 2.0 $\mathrm{eV})$, highlighting distinct surface electronic properties and density of states (DOS) of the two terminations. Specifically, we observe a zero-bias peak in the local DOS for the $\mathrm{PtTe}_{2}$ (Figure $1 \mathrm{~g}$ ) surface termination, while such a feature is absent on the $\mathrm{Pt}_{2} \mathrm{Te}_{2}$-terminated surface (Figure 1e).

Below we will show explicitly that the zero-bias peak arises from an electron pocket in the surface states of the $\mathrm{Pt}_{2} \mathrm{Te}_{2}$ terminated surface.

Bulk Band Structure and Topology. In order to further assess the dissimilarities in the surface electronic properties of the two terminations, we explored the electronic structure and spin texture of $\mathrm{Pt}_{3} \mathrm{Te}_{4}$. The bulk electronic band structure, without and with including the spin-orbit coupling (SOC) is shown in Figure 2a, b. The band structure clearly indicates the metallic character of the system. We find that the bands near the Fermi level predominantly arise from the Te $p$ states. The interplay between the different chalcogen $p$-derived states gives rise to a type-I bulk Dirac Fermion along the $\Gamma-Z$ direction of the Brillouin zone. This Dirac cone is also protected by the rotation symmetry, as confirmed by our symmetry analysis. The bands marked by $\Delta^{5,6}$ and $\Delta^{4}$ (see Figure $2 \mathrm{~b}$ ) have opposite rotation $\left(\mathrm{C}_{3}\right)$ characters $(-1$ and +1 , respectively) which prevents their hybridization and stabilizes the Dirac crossing. Other than this Dirac crossing, four different bands (marked explicitly in Figure 2b) cross the Fermi level, resulting in several hole and electron pockets. The Fermi surfaces corresponding to these four bands are shown in Figure $2 \mathrm{~d}$.

The bulk band structure of mitrofanovite displays multiple crossings between the valence and the conduction bands. This indicates the possibility of formation of topologically protected states. Furthermore, the $\Gamma^{4+}$ (band 3) and the $\Gamma^{4-}$ (band 4) bands are locally separated by a gap at every k-point. Therefore, to pin down the exact topology of this system, we computed the $\mathbb{Z}_{2}$ topological invariants. As $\mathrm{Pt}_{3} \mathrm{Te}_{4}$ preserves the inversion symmetry, the topological invariant is calculated using the parity-based method developed by Fu-Kane. ${ }^{25}$ The details of the number of occupied bands with a specific parity is presented in Table S1 of the Supporting Information. Based on the calculated parity of the occupied bands, the strong topological invariant $\left(\nu_{0}\right)$ is found to be zero and all the three weak topological invariants are nonzero. The four component $\mathbb{Z}_{2}$ invariant is found to be $(0 ; 111)$. As $\mathrm{Pt}_{3} \mathrm{Te}_{4}$ hosts Dirac cones, it has a Fermi surface like a metal, and it is 
a)

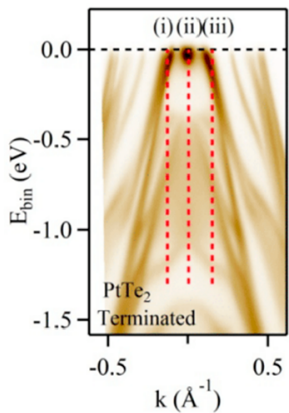

e) $\overline{\mathrm{K}} \quad \bar{\Gamma} \quad \overline{\mathrm{K}}$ b)

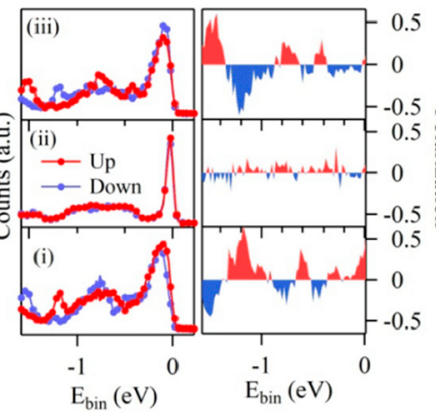

d)

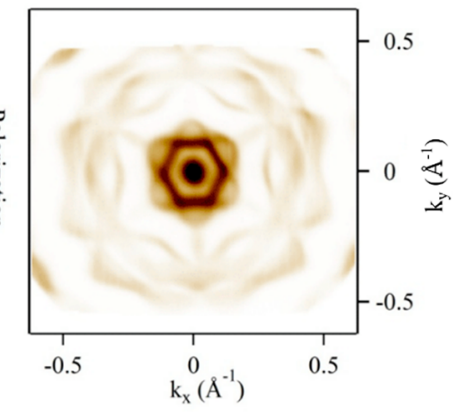

g)

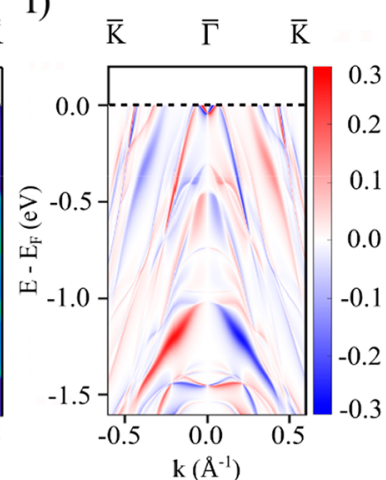

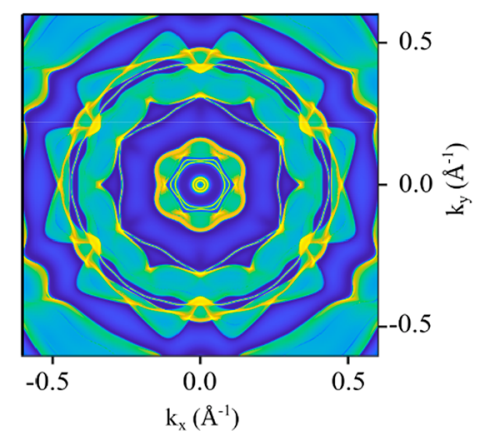

Figure 3. Experimental results and theoretical calculations for the $\mathrm{PtTe}_{2}$ termination. (a) Band dispersion along $\Gamma-\mathrm{K}$ measured with $h \nu=22$ eV. Red dotted lines indicate the positions in the momentum space where the spin spectra in part $b$ and the resulting spin polarization in part $\mathrm{c}$ were measured. All spin data are related to the component perpendicular to the momentum (i.e., perpendicular to $\Gamma-\mathrm{K}$ ), and there is a clear spin inversion between positive and negative momenta (i.e., between (i) and (iii)). (d) Measured Fermi surface.; (e) Calculated spectral function and (f) the corresponding spin texture. (g) Calculated Fermi surface.

a)

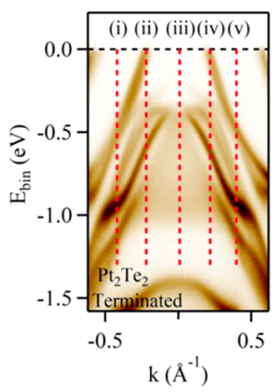

e) $\overline{\mathrm{K}}$

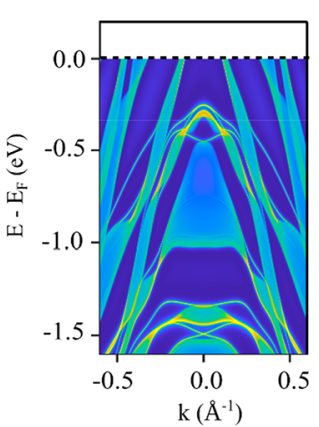

b)

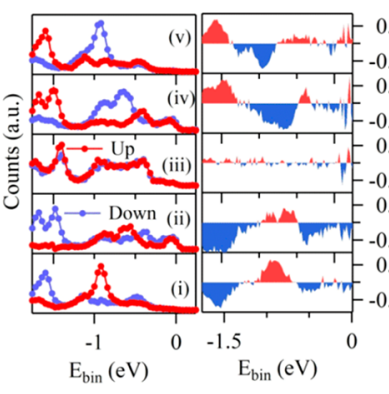

d)

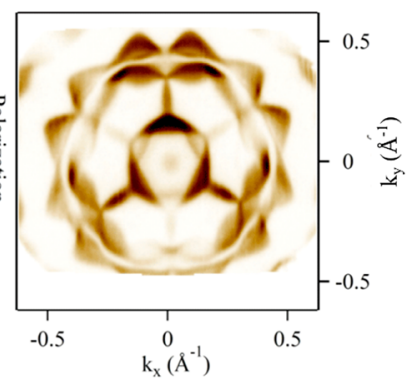

g)

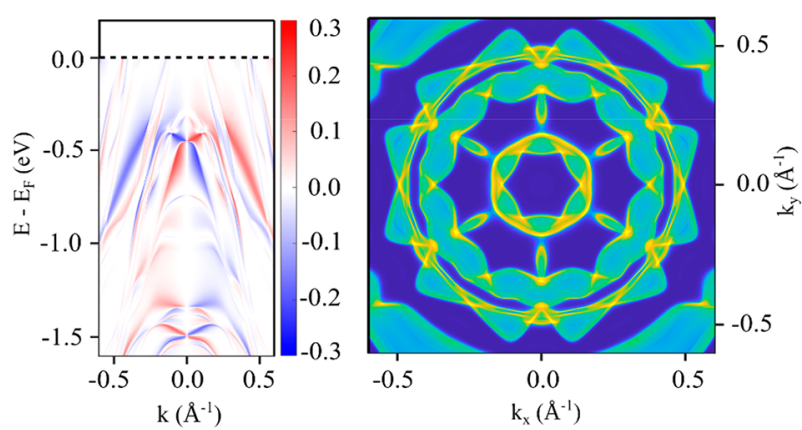

Figure 4. $\mathrm{Pt}_{2} \mathrm{Te}_{2}$ termination. Experimental results and theoretical calculations for the $\mathrm{Pt}_{2} \mathrm{Te}_{2}$ termination: (a) Band dispersion along the surface $\Gamma$-K direction measured with $h \nu=22 \mathrm{eV}$; red dotted lines indicate the positions in the momentum space where the spin spectra in part $b$ and the resulting spin polarization in part $c$ were measured; all spin data are related to the component perpendicular to the momentum (i.e., perpendicular to $\Gamma-K$ ), and there is a clear spin inversion between positive and negative momenta. (d) Measured Fermi surface. (e) Calculated spectral function. (f) Corresponding spin texture. (g) Calculated Fermi surface. 
characterized by a nontrivial topological index, which results in spin-polarized topological surface states, we classify it as a topological metal.

Spin-ARPES Results. The nontrivial topological invariant indicates the presence of topological surface states, displaying spin-momentum locking. To experimentally explore the topological surface states and their spin texture, we used spin- and angle-resolved photoemission spectroscopy (spinARPES).

Given the surface sensitivity of the technique (probing depth of the order of $10 \AA$ in our experimental conditions), we find that the measured electronic band structure is strongly termination dependent. This is evident in the two distinct data sets reported in Figures 3 and 4 obtained on the same sample just by changing the position between the two different terraces. The measured electronic dispersion along the surface $\Gamma-\mathrm{K}$ direction, for the $\mathrm{PtTe}_{2}$ and the $\mathrm{Pt}_{2} \mathrm{Te}_{2}$ terminations, is shown in Figures $3 \mathrm{a}$ and $4 \mathrm{a}$, respectively, with the corresponding Fermi surfaces reported in Figures $3 \mathrm{~d}$ and $4 \mathrm{~d}$. We ascribed those data to the two terminations by comparing the experimental results with the theoretically calculated surface band structure for a semi-infinite geometry terminating in either the $\mathrm{PtTe}_{2}$ or the $\mathrm{Pt}_{2} \mathrm{Te}_{2}$.

For the $\mathrm{PtTe}_{2}$ termination, we find that the surface spectral function has several bands, including the surface terminationinduced electron pocket at the center of the Brillouin zone (see Figure $3 \mathrm{a}$ and $3 \mathrm{e}$ ). In addition, we also identify four hole-like bands crossing the Fermi energy. Since these spectral features show almost no dependence on the photon energy (i.e., no $k_{z}$ dispersion is observed, see Supporting Information Figure S2), all of them can be ascribed to the two-dimensional surface states. The measured Fermi surface for the $\mathrm{PtTe}_{2}$ termination in Figure 3d clearly shows that the electron pocket at $\Gamma$ is surrounded by a hole pocket with 6 -fold rotation symmetry. The spin-polarization of these states is measured along three representative $k$ points ( $\Gamma$ and two symmetric points at positive and negative $k$ ) marked by dashed lines in Figure 3a. We acquired data for the spin in-plane direction perpendicular to the crystal momentum, typically indicative of spin-momentum locking.

The resulting spin resolved spectra and spin polarization are shown in Figure $3 b$ and $3 c$, respectively. While at $\Gamma$ (Figure 3b) the spin up (red) and spin down (blue) spectra are the same, resulting in zero spin polarization (Figure 3c), the situation is different for the two spectra taken at the two symmetric $k$ points around $\Gamma$. In fact, in panels (i) and (iii) the blue and red spectra are inverted, while the spin polarization is basically the same, but of opposite sign, in agreement with the spin-momentum locking. Note that not only the states at the Fermi energy exhibit spin polarization. It extends over the whole energy range probed in our experiment. All these features are well captured by our theoretical calculations for the semi-infinite geometry (see Figure $3 \mathrm{e}-\mathrm{g}$ ).

In contrast to the numerous bands crossing the Fermi energy for $\mathrm{PtTe}_{2}$, for $\mathrm{Pt}_{2} \mathrm{Te}_{2}$ termination only two evident Fermi crossings along the $\Gamma \mathrm{K}$ direction (Figure $4 \mathrm{a}$ ) exist. In general, for the $\mathrm{Pt}_{2} \mathrm{Te}_{2}$ termination, the number of electronic states below the Fermi energy is relatively lower as compared to the $\mathrm{PtTe}_{2}$-terminated surface. Additionally, the surface electron pocket at the $\Gamma$ point in the vicinity of the Fermi energy, observed for the $\mathrm{PtTe}_{2}$ termination, is absent for the $\mathrm{Pt}_{2} \mathrm{Te}_{2}$ termination (see Figure $4 \mathrm{a}$ ). These differences are also reflected in the zero-bias peak observed on the $\mathrm{PtTe}_{2}$ but not on $\mathrm{Pt}_{2} \mathrm{Te}_{2}$ terrace, where the number of states at the Fermi energy is relatively low. The theoretical Fermi surface plots for the two terminations indicate that both of them display a 6fold rotation symmetry (see Figures $3 \mathrm{~d}$ and Figure $4 \mathrm{~d}$ ). Similar to $\mathrm{PtTe}_{2}$ termination, also on $\mathrm{Pt}_{2} \mathrm{Te}_{2}$ we detect spin-polarized states (Figure $4 \mathrm{~b}$ and $\mathrm{c}$ ) with the polarization inversion across $\Gamma$ reflecting spin-momentum locking, evidenced as well in the calculated spin-resolved dispersion in Figure $4 \mathrm{f}$.

Independent of the band structure differences, for both the terminations the calculated spin-resolved electronic spectral function (see Figures $3 \mathrm{f}$ and $4 \mathrm{f}$ ) indicates that mitrofanovite is a strongly spin-orbit interacting system, with the surface states displaying spin-momentum locking. From the data in Figure $3 b-c$ and in Figure $4 b-c$, for the $\mathrm{PtTe}_{2}$ and $\mathrm{Pt}_{2} \mathrm{Te}_{2}$ terminations, respectively, we find that for both the terminations the spin polarization is zero at the $\Gamma$ point (Figure 3c, panel (ii), and Figure 4c, panel (iii). However, the spin polarization is finite and reaches up to $50 \%$ on the two sides of the $\Gamma$ point, with clear polarization inversion of the corresponding states (between panels (i)-(iii) for $\mathrm{PtTe}_{2}$ and between panels (i) $-(\mathrm{v})$ and (ii)-(iv) for the $\mathrm{Pt}_{2} \mathrm{Te}_{2}$ termination). Additionally, we observed spin-polarized states exhibiting spin-momentum locking over the full energy range that we probed. This observation is consistent with calculations for the spin-texture of the surface states in a finite-slab geometry (Figures $3 \mathrm{f}$ and $4 \mathrm{f}$ ). The large value of the measured spin polarization and the spin-momentum locking are also consistent with the topological origin of the observed surface states and the topological nature of $\mathrm{Pt}_{3} \mathrm{Te}_{4}$.

Interestingly, Pt-class type-II Dirac semimetal $\mathrm{PtTe}_{2}$ also hosts multiple topological surface states in a wide energy range, which arise from various band inversions within the Te porbital manifold. ${ }^{26,27}$ Similarly to $\mathrm{PtTe}_{2}, \mathrm{Pt}_{3} \mathrm{Te}_{4}$ exhibits topological surface states deep in the valence bands. However, there are significant differences in the surface states. For example, the Dirac-like surface state at the $\Gamma$ point observed for the $\mathrm{PtTe}_{2}$ termination in $\mathrm{Pt}_{3} \mathrm{Te}_{4}$ is absent in bulk $\mathrm{PtTe}_{2}$. Moreover, $\mathrm{PtTe}_{2}$ hosts an electron-pocket-like surface state near the Fermi level, which arises from the band inversions in the upper conduction bands, which are absent in $\mathrm{Pt}_{3} \mathrm{Te}_{4}$.

The observed surface states with robust spin polarization in $\mathrm{Pt}_{3} \mathrm{Te}_{4}$ can be exploited for making electrical devices for spininjection and spin-detection. ${ }^{28}$ Furthermore, the spin-momentum locked states in $\mathrm{Pt}_{3} \mathrm{Te}_{4}$ indicate that it can have a large spin-orbit torque. Thus, it can be used as a candidate in spintorque devices for applications in computation, logic, and memories. $^{28}$

Finally, as validated by atomic force microscopy (AFM) experiments in Supporting Information Figure S4, mitrofanovite-based surfaces also provide outstanding ambient stability even for prolonged storage in air. The chemical reactivity of both terminations is practically the same, as indicated by calculations reported in Table $S 1$ of the Supporting Information.

\section{CONCLUSIONS}

In summary, we have demonstrated that the recently discovered ambient-stable mitrofanovite $\mathrm{Pt}_{3} \mathrm{Te}_{4}$ is a topological metal with termination-dependent surface states and spin polarization using a combination of ARPES and STS experiments and $a b$ initio calculations. More interestingly, we find that the two distinct terminations $\left(\mathrm{PtTe}_{2}\right.$ and $\left.\mathrm{Pt}_{2} \mathrm{Te}_{2}\right)$ can arise as different terraces on the same face of the cleaved 
crystal. The two terminations have dissimilar electronic surface states, even though both terminations host spin-polarized surface states. The spin-polarized surface states display polarization reversal across the zone center, which is a characteristic feature of the spin-momentum locking. Our demonstration of termination-dependent electronic surface properties and spin polarization in $\mathrm{Pt}_{3} \mathrm{Te}_{4}$, combined with its excellent ambient stability, makes it an interesting candidate for exploring potential nanoelectronics, spintronics, optoelectronics, and plasmonic applications in mitrofanovite-based heterostructures and interfaces.

\section{METHODS}

Single-Crystal Growth. Single crystals of $\mathrm{Pt}_{3} \mathrm{Te}_{4}$ were grown by the self-flux method. Unlike the case of $\mathrm{PtTe}_{2},{ }^{29}$ the growth window of $\mathrm{Pt}_{3} \mathrm{Te}_{4}$ is narrow. The mixtures of high-purity $\mathrm{Pt}$ foil and $\mathrm{Te}$ ingots with the molar ratio of 51:49 were inserted in an alumina crucible and sealed into an evacuated quartz ampule. The quartz ampule was heated to $1080{ }^{\circ} \mathrm{C}$ for $24 \mathrm{~h}$ and then slowly cooled to $975^{\circ} \mathrm{C}$ at a rate of $1{ }^{\circ} \mathrm{C} / \mathrm{h}$. The excess flux was separated by centrifugation above 970 ${ }^{\circ} \mathrm{C}$ and mechanical polishing. Shiny platelike $\mathrm{Pt}_{3} \mathrm{Te}_{4}$ single crystals were harvested with a dimension of $4 \times 3 \times 0.4 \mathrm{~mm}^{3}$. The flat surface of the crystal corresponds to the (001) plane, as identified by XRD analyses reported in Figure S1 of the Supporting Information.

STM-ARPES. STM/STS were obtained with in situ STM/S (introduced in ref 30) on the same surfaces for which the ARPES data in Figures 3 and 4 were collected. The topographic images (Figure 1e and the right-side insets) were measured with constant current mode. The $\mathrm{dI} / \mathrm{dV}$ curves (Figures if and $\mathrm{lh}$ ) were numerically obtained from the measured I-V curves. All STM/STS experiments were carried out at room temperature.

Spin-ARPES experiments were carried out on the APE-LE beamline at Elettra synchrotron, described in ref 31 . We note here that with the available beam-spot size on the sample of $150 \times 50 \mu \mathrm{m}^{2}$, we could clearly distinguish between two different situations regarding the electronic band structures (as summarized in Figures 3 and 4) simply by moving the sample in front of the beam. This is feasible, considering that the size of terraces on as-cleaved $\mathrm{Pt}_{3} \mathrm{Te}_{4}$ surfaces exceeds $150 \mu \mathrm{m}^{2}$.

Computational Framework. First-principles calculations for the electronic properties were carried out using DFT, as implemented in the Vienna $a b$ initio Simulation Package (VASP). ${ }^{32,33}$ The exchangecorrelation effects were incorporated using the Perdew-BurkeErnzerhof (PBE) implementation of the generalized gradient approximation (GGA). ${ }^{34}$ A plane wave basis set with a cutoff energy of $500 \mathrm{eV}$ was used. The ionic relaxation was performed until the force on each atom was lower than $10^{-3} \mathrm{eV} / \AA$. The self-consistency convergence criteria for the total energies were set to $10^{-7} \mathrm{eV}$, and a $\Gamma$-centered $8 \times 8 \times 8$ Monkhorst-Pack $k$-point grid $^{35}$ was used to perform the Brillouin zone integration. We constructed the tight binding model with the atom centered "Wannier-like" orbitals using the VASP2WANNIER $90^{36}$ interface. A surface energy spectrum was obtained within the iterative Green's function method following the implementation in the WannierTools package. ${ }^{37}$

\section{ASSOCIATED CONTENT}

\section{(1) Supporting Information}

The Supporting Information is available free of charge at https://pubs.acs.org/doi/10.1021/acsnano.1c04766.

Additional information about (i) experimental characterization, (ii) the topological invariants, (iii) ARPES measurements, and (iv) ambient stability of $\mathrm{Pt}_{3} \mathrm{Te}_{4}$ (PDF)

\section{AUTHOR INFORMATION}

\section{Corresponding Authors}

Ivana Vobornik - CNR-IOM, TASC Laboratory, Area Science Park-Basovizza, 34139 Trieste,, Italy;

Email: ivana.vobornik@elettra.eu

Amit Agarwal - Department of Physics, Indian Institute of Technology Kanpur, Kanpur 208016, India;

Email: amitag@iitk.ac.in

Antonio Politano - INSTM and Department of Physical and Chemical Sciences, University of L'Aquila, via Vetoio 67100 L'Aquila (AQ), Italy; CNR-IMM Istituto per la Microelettronica e Microsistemi, I-95121 Catania, Italy; (1) orcid.org/0000-0002-4254-2102; Email: antonio.politano@univaq.it

\section{Authors}

Jun Fujii - CNR-IOM, TASC Laboratory, Area Science ParkBasovizza, 34139 Trieste, Italy

Barun Ghosh - Department of Physics, Indian Institute of Technology Kanpur, Kanpur 208016, India

Anan Bari Sarkar - Department of Physics, Indian Institute of Technology Kanpur, Kanpur 208016, India

Debashis Mondal - CNR-IOM, TASC Laboratory, Area Science Park-Basovizza, 34139 Trieste,, Italy

Chia-Nung Kuo - Department of Physics, National Cheng Kung University, 70101 Tainan, Taiwan; Taiwan Consortium of Emergent Crystalline Materials, Ministry of Science and Technology, Taipei 10601, Taiwan

François C. Bocquet - Peter Grünberg Institut (PGI-3), Forschungszentrum Jülich, 52425 Jülich, Germany; Jülich Aachen Research Alliance (JARA), Fundamentals of Future Information Technology, 52425 Jülich, Germany; (1) orcid.org/0000-0002-9471-4439

Lixue Zhang - College of Chemistry and Chemical Engineering, Qingdao University, Qingdao 266071 Shandong, P. R. China; (1) orcid.org/0000-0003-3430-4988

Danil W. Boukhvalov - College of Science, Institute of Materials Physics and Chemistry, Nanjing Forestry University, Nanjing 210037, P. R. China; Institute of Physics and Technology, Ural Federal University, 620002 Ekaterinburg, Russia; (i) orcid.org/0000-0002-2286-3443

Chin Shan Lue - Department of Physics, National Cheng Kung University, 70101 Tainan, Taiwan; Taiwan Consortium of Emergent Crystalline Materials, Ministry of Science and Technology, Taipei 10601, Taiwan

Complete contact information is available at:

https://pubs.acs.org/10.1021/acsnano.1c04766

\section{Author Contributions}

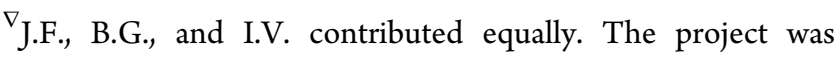
conceived and coordinated by A.P. STM/STS experiments were carried out by J.F. The theoretical model was elaborated by B.G., A.B.S., and A.A. LEED experiments were carried out by F.C.B. and A.P. Spin-ARPES and XPS experiments were performed by D.M., J.F., and I.V. Ambient stability was addressed by D.W.B., L.Z., and A.P. Samples were grown by C.N.K., C.S.L., and A.P. The paper was written by J.F., I.V., B.G., A.A., and A.P.

\section{Notes}

The authors declare no competing financial interest. 


\section{ACKNOWLEDGMENTS}

This work has been partly performed in the framework of the nanoscience foundry and fine analysis (NFFA-MUR Italy Progetti Internazionali) facility. L.Z. acknowledges funding by the National Natural Science Foundation of China (No. 21775078). F.C.B. acknowledges funding by the DFG through the SFB 1083 Structure and Dynamics of Internal Interfaces (Project A 12). D.W.B. acknowledges support by Ministry of Science and Higher Education of the Russian Federation (through the basic part of the government mandate, Project No. FEUZ-2020-0060) and Jiangsu Innovative and Entrepreneurial Talents Project. B.G. and A.A. acknowledge funding from Science and Engineering Research Board (SERB) and Department of Science and Technology (DST), government of India. A.A. thanks the HPC facility at IIT Kanpur for computational resources. C.S.L. and C.N.K. acknowledge funding by the Ministry of Science and Technology of Taiwan (MOST-110-2124-M-006-010).

\section{REFERENCES}

(1) Breitkreiz, M.; Brouwer, P. W. Large Contribution of Fermi Arcs to the Conductivity of Topological Metals. Phys. Rev. Lett. 2019, 123 (6), 066804.

(2) Hosen, M. M.; Dimitri, K.; Nandy, A. K.; Aperis, A.; Sankar, R.; Dhakal, G.; Maldonado, P.; Kabir, F.; Sims, C.; Chou, F.; Kaczorowski, D.; Durakiewicz, T.; Oppeneer, P. M.; Neupane, M. Distinct Multiple Fermionic States in a Single Topological Metal. Nat. Commun. 2018, 9 (1), 3002.

(3) Kumar, N.; Sun, Y.; Nicklas, M.; Watzman, S. J.; Young, O.; Leermakers, I.; Hornung, J.; Klotz, J.; Gooth, J.; Manna, K.; Süß, V.; Guin, S. N.; Förster, T.; Schmidt, M.; Muechler, L.; Yan, B.; Werner, P.; Schnelle, W.; Zeitler, U.; Wosnitza, J.; Parkin, S. S. P.; Felser, C.; Shekhar, C. Extremely High Conductivity Observed in the Triple Point Topological Metal MoP. Nat. Commun. 2019, 10 (1), 2475.

(4) Li, Y.; Haldane, F. D. M. Topological Nodal Cooper Pairing in Doped Weyl Metals. Phys. Rev. Lett. 2018, 120 (6), 067003.

(5) Nayak, J.; Wu, S. C.; Kumar, N.; Shekhar, C.; Singh, S.; Fink, J.; Rienks, E. E. D.; Fecher, G. H.; Parkin, S. S. P.; Yan, B.; Felser, C. Multiple Dirac Cones at the Surface of the Topological Metal LaBi. Nat. Commun. 2017, 8, 13942.

(6) Shao, D. F.; Gurung, G.; Zhang, S. H.; Tsymbal, E. Y. Dirac Nodal Line Metal for Topological Antiferromagnetic Spintronics. Phys. Rev. Lett. 2019, 122 (7), 077203.

(7) Wang, Z.; Gresch, D.; Soluyanov, A. A.; Xie, W.; Kushwaha, S.; Dai, X.; Troyer, M.; Cava, R. J.; Bernevig, B. A. MoTe 2 : A Type-II Weyl Topological Metal. Phys. Rev. Lett. 2016, 117 (5), 056805.

(8) Ortiz, B. R.; Teicher, S. M.; Hu, Y.; Zuo, J. L.; Sarte, P. M.; Schueller, E. C.; Abeykoon, A. M.; Krogstad, M. J.; Rosenkranz, S.; Osborn, R. $\mathrm{CsV}_{3} \mathrm{Sb}_{5}$ : A $\mathrm{Z}_{2}$ Topological Kagome Metal with a Superconducting Ground State. Phys. Rev. Lett. 2020, 125 (24), 247002.

(9) Li, S.; Yu, Z.-M.; Yao, Y.; Yang, S. A. Type-II Topological Metals. Frontiers of Physics 2020, 15 (4), 43201.

(10) Politano, A.; Chiarello, G.; Ghosh, B.; Sadhukhan, K.; Kuo, C.N.; Lue, C. S.; Pellegrini, V.; Agarwal, A. 3D Dirac Plasmons in the Type-II Dirac Semimetal PtTe 2 . Phys. Rev. Lett. 2018, 121 (8), 086804.

(11) Armitage, N.; Mele, E.; Vishwanath, A. Weyl and Dirac Semimetals in Three-Dimensional Solids. Rev. Mod. Phys. 2018, 90 (1), 015001.

(12) Bansil, A.; Lin, H.; Das, T. Colloquium: Topological Band Theory. Rev. Mod. Phys. 2016, 88 (2), 021004.

(13) Guan, S.-Y.; Chen, P.-J.; Chu, M.-W.; Sankar, R.; Chou, F.; Jeng, H.-T.; Chang, C.-S.; Chuang, T.-M. Superconducting Topological Surface States in the Noncentrosymmetric Bulk Superconductor $\mathrm{PbTaSe}_{2}$. Sci. Adv. 2016, 2 (11), e1600894.
(14) Sato, M.; Ando, Y. Topological Superconductors: a Review. Rep. Prog. Phys. 2017, 80 (7), 076501.

(15) Yang, Q.; Li, G.; Manna, K.; Fan, F.; Felser, C.; Sun, Y. Topological Engineering of Pt-Group-Metal-Based Chiral Crystals toward High-Efficiency Hydrogen Evolution Catalysts. Adv. Mater. 2020, 32 (14), 1908518.

(16) Xiao, Z.; Laplante, A. Characterizing and Recovering the Platinum Group Minerals--A Review. Miner. Eng. 2004, 17 (9-10), 961-979.

(17) Bahramy, M. S.; Clark, O. J.; Yang, B. J.; Feng, J.; Bawden, L.; Riley, J. M.; Marković, I.; Mazzola, F.; Sunko, V.; Biswas, D.; Cooil, S. P.; Jorge, M.; Wells, J. W.; Leandersson, M.; Balasubramanian, T.; Fujii, J.; Vobornik, I.; Rault, J. E.; Kim, T. K.; Hoesch, M.; Okawa, K.; Asakawa, M.; Sasagawa, T.; Eknapakul, T.; Meevasana, W.; King, P. D. C. Ubiquitous Formation of Bulk Dirac Cones and Topological Surface States from a Single Orbital Manifold in Transition-Metal Dichalcogenides. Nat. Mater. 2018, 17 (1), 21-28.

(18) D'Olimpio, G.; Guo, C.; Kuo, C.-N.; Edla, R.; Lue, C. S.; Ottaviano, L.; Torelli, P.; Wang, L.; Boukhvalov, D. W.; Politano, A. $\mathrm{PdTe}_{2}$ Transition-Metal Dichalcogenide: Chemical Reactivity, Thermal Stability and Device Implementation. Adv. Funct. Mater. 2020, 30 (5), 1906556.

(19) Xu, H.; Guo, C.; Zhang, J.; Guo, W.; Kuo, C. N.; Lue, C. S.; Hu, W.; Wang, L.; Chen, G.; Politano, A.; Chen, X.; Lu, W. PtTe $2^{-}$ Based Type-II Dirac Semimetal and Its van der Waals Heterostructure for Sensitive Room Temperature Terahertz Photodetection. Small 2019, 15 (52), 1903362.

(20) Kireev, D.; Okogbue, E.; Jayanth, R. T.; Ko, T.-J.; Jung, Y.; Akinwande, D. Multipurpose and Reusable Ultrathin Electronic Tattoos Based on $\mathrm{PtSe}_{2}$ and $\mathrm{PtTe}_{2}$. ACS Nano 2021, 15 (2), 28002811.

(21) Subbotin, V. V.; Vymazalová, A.; Laufek, F.; Savchenko, Y. E.; Stanley, C. J.; Gabov, D. A.; Plásil, J. Mitrofanovite, $\mathrm{Pt}_{3} \mathrm{Te}_{4}$, a New Mineral from the East Chuarvy Deposit, Fedorovo-Pana Intrusion, Kola Peninsula, Russia. Mineral. Mag. 2019, 83 (4), 523-530.

(22) Makvandi, S.; Pagé, P.; Tremblay, J.; Girard, R. Exploration for Platinum-Group Minerals in Till: A New Approach to the Recovery, Counting, Mineral Identification and Chemical Characterization. Minerals 2021, 11 (3), 264.

(23) Bae, D.; Park, K.; Kwon, H.; Won, D.; Ling, N.; Baik, H.; Yang, J.; Park, H. J.; Cho, J.; Yang, H. Mitrofanovite, Layered Platinum Telluride, for Active Hydrogen Evolution. ACS Appl. Mater. Interfaces 2021, 13 (2), 2437-2446.

(24) Tanuma, S.; Powell, C. J.; Penn, D. R. Calculations of Electron Inelastic Mean Free Paths (IMFPS). IV. Evaluation of Calculated IMFPs and of the Predictive IMFP Formula TPP-2 for Electron Energies Between 50 and 2000 eV. Surf. Interface Anal. 1993, 20 (1), $77-89$.

(25) Kane, C. L.; Mele, E. J. Quantum Spin Hall Effect in Graphene. Phys. Rev. Lett. 2005, 95 (22), 226801.

(26) Yan, M.; Huang, H.; Zhang, K.; Wang, E.; Yao, W.; Deng, K.; Wan, G.; Zhang, H.; Arita, M.; Yang, H. Lorentz-Violating Type-II Dirac Fermions in Transition Metal Dichalcogenide $\mathrm{PtTe}_{2}$. Nat. Commun. 2017, 8 (1), 257.

(27) Ghosh, B.; Mondal, D.; Kuo, C.-N.; Lue, C. S.; Nayak, J.; Fujii, J.; Vobornik, I.; Politano, A.; Agarwal, A. Observation of Bulk States and Spin-Polarized Topological Surface States in Transition Metal Dichalcogenide Dirac Semimetal Candidate $\mathrm{NiTe}_{2}$. Phys. Rev. B 2019, 100 (19), 195134.

(28) Tang, J.; Chang, L.-T.; Kou, X.; Murata, K.; Choi, E. S.; Lang, M.; Fan, Y.; Jiang, Y.; Montazeri, M.; Jiang, W.; Wang, Y.; He, L.; Wang, K. L. Electrical Detection of Spin-Polarized Surface States Conduction in $\left(\mathrm{Bi}_{0.53} \mathrm{Sb}_{0.47}\right)_{2} \mathrm{Te}_{3}$ Topological Insulator. Nano Lett. 2014, 14 (9), 5423-5429.

(29) Dey, S.; Jain, V. K. Platinum Group Metal Chalcogenides. Platinum Metals Review 2004, 48 (1), 16-28.

(30) Panaccione, G.; Vobornik, I.; Fujii, J.; Krizmancic, D.; Annese, E.; Giovanelli, L.; MacCherozzi, F.; Salvador, F.; De Luisa, A.; Benedetti, D.; Gruden, A.; Bertoch, P.; Polack, F.; Cocco, D.; Sostero, 
G.; Diviacco, B.; Hochstrasser, M.; Maier, U.; Pescia, D.; Back, C. H.; Greber, T.; Osterwalder, J.; Galaktionov, M.; Sancrotti, M.; Rossi, G. Advanced Photoelectric Effect Experiment Beamline at Elettra: A Surface Science Laboratory Coupled with Synchrotron Radiation. Rev. Sci. Instrum. 2009, 80 (4), 043105.

(31) Bigi, C.; Das, P. K.; Benedetti, D.; Salvador, F.; Krizmancic, D.; Sergo, R.; Martin, A.; Panaccione, G.; Rossi, G.; Fujii, J. Very Efficient Spin Polarization Analysis (VESPA): New Exchange Scattering-Based Setup for Spin-Resolved ARPES at APE-NFFA Beamline at Elettra. J. Synchrotron Radiat. 2017, 24 (4), 750-756.

(32) Kresse, G.; Furthmüller, J. Efficient Iterative Schemes for $a b$ Initio Total-Energy Calculations Using a Plane-Wave Basis Set. Phys. Rev. B 1996, 54 (16), 11169-11186.

(33) Kresse, G.; Joubert, D. From Ultrasoft Pseudopotentials to the Projector Augmented-Wave Method. Phys. Rev. B: Condens. Matter Mater. Phys. 1999, 59 (3), 1758.

(34) Perdew, J. P.; Burke, K.; Ernzerhof, M. Generalized Gradient Approximation Made Simple. Phys. Rev. Lett. 1996, 77 (18), 38653868.

(35) Monkhorst, H. J.; Pack, J. D. Special Points for Brillouin-Zone Integrations. Phys. Rev. B 1976, 13 (12), 5188-5192.

(36) Marzari, N.; Vanderbilt, D. Maximally Localized Generalized Wannier Functions for Composite Energy Bands. Phys. Rev. B 1997, $56(20), 12847$.

(37) Wu, Q.; Zhang, S.; Song, H.-F.; Troyer, M.; Soluyanov, A. A. WannierTools: An Open-Source Software Package for Novel Topological Materials. Comput. Phys. Commun. 2018, 224, 405-416. 